\title{
Oxidative phosphorylation and lacunar stroke
}

\author{
Genome-wide enrichment analysis of common variants \\ OPEN
}

Matthew Traylor, $\mathrm{PhD}^{*}$

Christopher D. Anderson,

$\mathrm{MD}, \mathrm{MMSc}^{*}$

Robert Hurford, BMBS, MSc

Steve Bevan, $\mathrm{PhD}$

Hugh S. Markus, DM

Correspondence to

Dr. Traylor:

mt628@medschl.cam.ac.uk
Supplemental data at Neurology.org

\section{ABSTRACT}

Objective: We investigated whether oxidative phosphorylation (OXPHOS) abnormalities were associated with lacunar stroke, hypothesizing that these would be more strongly associated in patients with multiple lacunar infarcts and leukoaraiosis (LA).

Methods: In 1,012 MRI-confirmed lacunar stroke cases and 964 age-matched controls recruited from general practice surgeries, we investigated associations between common genetic variants within the OXPHOS pathway and lacunar stroke using a permutation-based enrichment approach. Cases were phenotyped using MRI into those with multiple infarcts or LA (MLI/LA) and those with isolated lacunar infarcts (ILI) based on the number of subcortical infarcts and degree of LA, using the Fazekas grading. Using gene-level association statistics, we tested for enrichment of genes in the OXPHOS pathway with all lacunar stroke and the 2 subtypes.

Results: There was a specific association with strong evidence of enrichment in the top 1\% of genes in the MLI/LA (subtype $p=0.0017$ ) but not in the ILI subtype $(p=1)$. Genes in the top percentile for the all lacunar stroke analysis were not significantly enriched ( $p=0.07$ ).

Conclusions: Our results implicate the OXPHOS pathway in the pathogenesis of lacunar stroke, and show the association is specific to patients with the MLI/LA subtype. They show that MRIbased subtyping of lacunar stroke can provide insights into disease pathophysiology, and imply that different radiologic subtypes of lacunar stroke subtypes have distinct underlying pathophysiologic processes. Neurology ${ }^{\circledR} 2016 ; 86: 141-145$

\section{GLOSSARY}

GSEA = gene-set enrichment analysis algorithm; ILI = isolated lacunar infarct; LA = leukoaraiosis; LD = linkage disequilibrium; MELAS = mitochondrial encephalomyopathy, lactic acidosis, and stroke-like episodes; $\mathbf{M L I}$ = multiple lacunar infarcts; NO = nitric oxide; OXPHOS = oxidative phosphorylation; SNP = single nucleotide polymorphism

Accumulating evidence suggests that changes in mitochondrial function influence risk of stroke, particularly for the ischemic and hemorrhagic stroke subtypes arising from cerebral small vessel disease. ${ }^{1-3}$ The mitochondrial genome is essential for the assembly of the oxidative phosphorylation (OXPHOS) apparatus, consisting of 5 protein complexes necessary for maintenance of aerobic haemostasis. ${ }^{4}$ Genetic variants lying within genes encoding the OXPHOS apparatus, the majority of which reside in the autosome, have been associated with risk of lacunar stroke and deep intracerebral hemorrhage. ${ }^{1-3}$ Additionally, multiple rare disorders are caused by mutations in OXPHOS genes, ${ }^{5}$ many of which result in stroke-like episodes, neurodegeneration, and leukoencephalopathies.

Neuropathologic studies suggest that lacunar stroke results from a number of differing vascular pathologies, including focal atherosclerosis, often associated with isolated larger lacunar infarcts, and a more diffuse arteriopathy, usually seen in hypertensive individuals. ${ }^{6}$ The diffuse arteriopathy has been associated both pathologically and neuroradiologically with multiple lacunar infarcts, as well as confluent leukoaraiosis (LA) on MRI. ${ }^{7}$ Given the considerations described above, one

\footnotetext{
*These authors contributed equally to this work.

From Clinical Neurosciences (M.T., R.H., H.S.M.), University of Cambridge, UK; School of Life Science (S.B.), University of Lincoln, UK; and the Center for Human Genetic Research (C.D.A.), Department of Neurology, Massachusetts General Hospital, Boston.

Go to Neurology.org for full disclosures. Funding information and disclosures deemed relevant by the authors, if any, are provided at the end of the article. The Article Processing Charge was paid by Wellcome Trust.

This is an open access article distributed under the terms of the Creative Commons Attribution License 4.0 (CC BY), which permits unrestricted use, distribution, and reproduction in any medium, provided the original work is properly cited.
} 
might hypothesize that genetic variation within OXPHOS is more likely to be associated with patients with LA or multiple infarcts.

We investigated whether common genetic variants within the OXPHOS pathway were involved in the pathogenesis of MRI-confirmed lacunar stroke using a permutation-based enrichment approach, evaluating the strength of genetic associations within genes in the OXPHOS complex compared to the background of random genes across the autosome, and determining whether strength of any associations differed by lacunar stroke subtype.

METHODS Study population. A total of 1,029 Caucasian patients with lacunar stroke, aged $\leq 70$ years, were recruited from 72 specialist stroke centers throughout the United Kingdom (supplementary material on the Neurology ${ }^{\circledR}$ Web site at Neurology.org), between 2002 and 2012, as part of the Young Lacunar Stroke DNA Resource. Lacunar stroke was defined as a clinical lacunar syndrome, ${ }^{8}$ with an anatomically compatible lesion on MRI seen as either acute on diffusion-weighted imaging or low signal on $\mathrm{T} 1$ or fluid-attenuated inversion recovery imaging and with diameter $\leq 15 \mathrm{~mm}$. All patients underwent full stroke investigation, including brain MRI, imaging of the extracerebral arteries, arteries, and ECG. Echocardiography was performed when appropriate. All MRIs and clinical histories were reviewed centrally by one physician (H.S.M.). Exclusion criteria were as follows: stenosis $>50 \%$ in the extracranial or intracranial cerebral vessels or previous carotid endarterectomy; cardioembolic source of stroke, defined according to the Trial of Org 10172 in Acute Stroke Treatment criteria9 as high or moderate probability; cortical infarct on MRI; subcortical infarct $>15 \mathrm{~mm}$ in diameter, as these can be caused by embolic mechanisms (striatocapsular infarcts); and any other specific cause of stroke (e.g., lupus anticoagulant, cerebral vasculitis, dissection, monogenic cause of stroke). All cases were screened for NOTCH3 cerebral autosomal dominant arteriopathy with subcortical infarcts and leukoencephalopathy and Fabry disease mutations and positive cases were excluded. An additional 82 Caucasian patients with lacunar stroke were recruited from St. George's Hospital, London, as part of the GENESIS study. The same inclusion and exclusion criteria were used as in the DNA-lacunar study except that older patients were also included, and a similar investigation protocol was used with all patients having brain MRI.

Unrelated Caucasian controls, free of clinical cerebrovascular disease, were obtained by random sampling from general practice lists from 4 sites in England and Scotland and chosen to match the same geographical location as the patients. Sampling was stratified for age and sex. All patients and controls underwent a standardized clinical assessment and completed a standardized study questionnaire with the same risk factor definitions as used for the cases. MRI was not performed in controls.

Standard protocol approvals, registrations, and patient consents. The study was approved by the Multi-Centre Research Ethics Committee (04/MRE00/36) and informed consent was obtained from all participants.

Subtyping of lacunar stroke. We used the Fazekas scale, a semiquantitative rating scale that classifies LA into 4 groups ranging from none (0) to severe (3), to grade the extent of LA on MRI.
Based on this grading, we separated the patients into 2 strata: (1) isolated lacunar infarct (ILI): patients with only a single infarct and mild or absent LA (Fazekas grade $<2$ ); (2) multiple lacunar infarcts (MLI)/LA: MLI or moderate to severe LA (Fazekas grade $\geq 2$ ). We randomly selected 20 MRI scans to be graded for a second time by the same rater. Perfect agreement was found in assignment to either of the 2 groups $(\kappa=1)$. In addition, to evaluate inter-rater reliability, we randomly selected 40 scans, which were graded by a second rater (R.H.). Perfect agreement was found between the 2 raters for allocation of lacunar subtype $(\kappa=1)$. However, one scan was graded MLI by one rater and LA by the other.

Genotyping and imputation. All included samples were genotyped on the Illumina (San Diego, CA) HumanExomeCore array. Single nucleotide polymorphisms (SNPs) were excluded if they had minor allele frequency $<0.01$, had genotype missingness $>3 \%$, had Hardy-Weinberg equilibrium $p<1 \mathrm{e}-6$ in controls, were strand ambiguous (A/T or $\mathrm{C} / \mathrm{G}$ ), or showed evidence of differential missingness by case-control status $(p<0.05)$. Individuals were excluded if they had missingness $>3 \%$, had excess or reduced heterozygosity, showed evidence of relatedness with another individual (pi-hat $>0.1875$ ), or failed a sex check in PLINK. To assess population ancestry, all individuals were merged with the HapMap II populations (CEU, YRI, JPT + CHB). The merged dataset was linkage disequilibrium (LD) pruned and regions with long-range LD were removed. Ancestry-informative principal components were determined using EIGENSTRAT's smartpca function. ${ }^{10} \mathrm{~A}$ total of 284 individuals were removed who did not segregate with CEU HapMap II individuals. We then repeated smartpca on the remaining Caucasian individuals. We removed all individuals who were more than 6 standard deviations from the mean on the first 2 principal components of the first 8 iterations of smartpca. The remaining 269,691 autosomal SNPs and 2,603 individuals were then imputed to 1000 Genomes phase 1 (March 2012) ${ }^{11}$ : SHAPEIT v2 was used to phase the haplotypes and IMPUTE v2.2.2 was used to perform the imputation, ${ }^{12,13}$ resulting in 9,289,526 SNPs.

Enrichment analysis. We first tested genome-wide association with each autosomal SNP, including the first 2 principal components as covariates, using SNPTEST v.2.4. From the genome-wide association statistics, we calculated gene-level statistics for each autosomal gene using the VEGAS software package. ${ }^{14}$ VEGAS simulates SNP associations based on the LD structure of each gene $( \pm 50 \mathrm{~Kb}$ window $)$ and calculates an empirical $p$ value for association of each gene with disease status based on the proportion of simulations in which the sum of simulated SNP $\chi^{2}$ statistics exceeds the observed sum from the association results.

We then used the generated gene-level statistics to test for significant enrichment of the OXPHOS genes compared to random sets of genes of the same number. We obtained the gene-level results (from VEGAS) for all OXPHOS genes, and tabulated the number of genes from the pathway in the top $1 \%, 5 \%$, and $10 \%$ of all genes. We then generated 1,000 gene sets of the same length, sampling randomly from all gene level results from VEGAS (increased to 10,000 permutations for $p<0.05$ ). We calculated significance by determining the proportion of randomly permuted gene sets in which as many or more genes were from the given percentile of all genes. In addition, we used the gene-set enrichment analysis algorithm (GSEA) to test for overall enrichment across all genes, simulating as previously. ${ }^{15}$ We first performed all analyses for all lacunar stroke cases vs controls, and then for the 2 subtypes of ILI or MLI/LA vs controls (figure 1). We set a significance threshold of $p=0.0042$, corresponding to Bonferroni correction for the 12 analyses (3 phenotypes, 4 tests). 
Figure 1 Flowchart of analyses performed

GWAS dataset of 1,012 MRI confirmed lacunar stroke cases and 964 controls; cases subtyped into MLI/LA and ILI

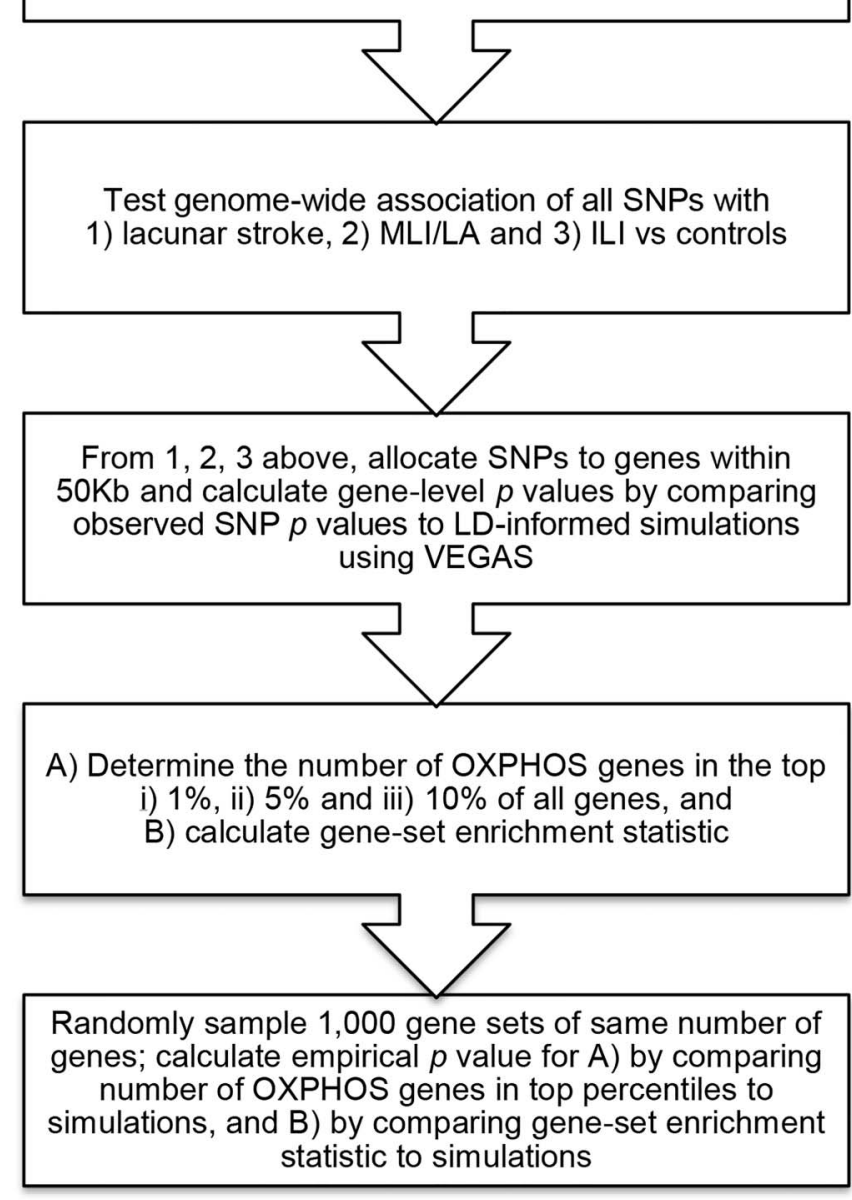

GWAS = genome-wide association study; ILI = isolated lacunar infarct; LA = leukoaraiosis; $\mathrm{LD}=$ linkage disequilibrium; $\mathrm{MLI}=$ multiple lacunar infarcts; $\mathrm{OXPHOS}=$ oxidative phosphorylation pathway; SNP = single nucleotide polymorphism.

RESULTS Details of the study population are given in table 1; 1,012 cases and 964 controls remained after genotyping quality control steps, with 502 cases in the MLI/LA subtypes and 501 in the ILI subtype; in 9 cases, clear separation into different phenotypes was not possible based on brain imaging. We calculated gene-based statistics for 17,656 autosomal genes in each of the analyses using the VEGAS package. ${ }^{14}$ Using these gene-level association statistics, we tested for enrichment of genes in the OXPHOS pathway with lacunar stroke. We found a near-significant enrichment $(p=0.07)$ of genes in the top percentile with all lacunar stroke analysis (table 2). We then performed secondary analyses in the MLI/LA and ILI subtypes. This showed strong evidence of enrichment in the top $1 \%$ of genes with the MLI/LA subtype $(p=0.0017)$.
Conversely, we found no association with the ILI subtype $(p=1)$. The OXPHOS genes in the top $1 \%$ for the MLI/LA subtyped consisted of NDUFB2, SURF1, UQCRH, NDUFS1, and ATP5I. Plots of the $p$ values in these gene regions by genomic position are provided in figure e-1. We followed up our significant result with MLI/LA by testing for enrichment in any of the OXPHOS subcomplexes (table 2). However, we could find no evidence that enrichment was specific to any subcomplex. Indeed, the 5 OXPHOS genes in the top $1 \%$ for MLI/LA were divided between OXPHOS subcomplexes: 2 were in complex I (NDUFB2, NDUFS1), 1 was in complex II $(U Q C R H), 1$ was in complex IV (SURF1), and 1 was in complex V (ATP5I). We found no significant enrichment in the top $5 \%$ or $10 \%$ of genes, or when using the GSEA algorithm (table 2).

DISCUSSION In this genome-wide enrichment analysis of MRI-confirmed lacunar stroke cases and age-matched controls, we evaluated an association of the oxidative phosphorylation pathway with lacunar stroke, finding an association in cases with LA or multiple lacunar infarcts, with no evidence for association in cases with isolated lacunar infarcts. The present study extends the previous findings by Anderson et al., ${ }^{3}$ and defines the phenotypes driving this previously reported genetic association.

Although our approach highlights an aggregate association and therefore cannot identify specific causal genetic variants, these results have important implications. First, they provide evidence for the association of the OXPHOS pathway with lacunar stroke, and importantly show that the association is specific and limited to a subgroup of SVD patients, namely those with multiple lacunar infarcts/LA. Potential mechanisms by which OXPHOS dysfunction increases risk of lacunar stroke are numerous, including depletion of ATP, generation of reactive oxygen species, alterations in autophagy, or changes to cell signaling. Mitochondrial dysfunction and oxidative stress in endothelial cells have been shown to result in increased blood-brain barrier permeability, as well as to increase the expression of adhesion molecules. ${ }^{16,17}$ One intriguing hypothesis is that mitochondrial dysfunction leads to endothelial dysfunction through nitric oxide (NO) dysregulation. A similar effect has been seen in the mitochondrial encephalomyopathy, lactic acidosis, and stroke-like episodes (MELAS), where citrulline and arginine supplementations have shown improved outcomes by ameliorating NO production. ${ }^{18,19}$ Given that white 
Table 1 Cohort characteristics

$\begin{array}{lllll} & \text { All lacunar } & \text { MLI/LA subtype } & \text { ILI subtype } & \text { Controls } \\ \text { No. } & 1,012 & 502 & 501 & 964 \\ \text { Age, y, mean (SD) } & 57.2(9.6) & 59.5(9.1) & 54.7(9.2) & 59.7(4.3) \\ \text { Male, \% } & 69.8 & 71.6 & 68.3 & 52.5 \\ \text { Hypertension, \% } & 70.8 & 79.2 & 62.9 & 53.3 \\ \text { Diabetes, \% } & 16.7 & 19.0 & 14.2 & 7.7 \\ \text { Hyperlipidaemia, \% } & 68.1 & 70.6 & 65.4 & 58.1 \\ \text { Ever smoker, \% } & 69.6 & 75.0 & 64.1 & 56.0 \\ \text { Fazekas LA grade, mean (SD) } & 0.96(1.07) & 1.72(1.00) & 0.21(0.42) & \mathrm{NA}\end{array}$

Abbreviations: ILI = isolated lacunar infarct; $\mathrm{LA}=$ leukoaraiosis; $\mathrm{MLI}=$ multiple lacunar infarcts; NA = not applicable.

matter disease imaging phenotypes are present in both MELAS and our MLI/LA cohort, this latter mechanism may deserve further scrutiny in functional follow-up studies building on these results.

Second, our results highlight the importance of careful subtyping of stroke cases, demonstrating that detailed phenotyping with MRI can provide valuable insights into disease pathogenesis. In this case, subtyping lacunar strokes using a classification scheme supported by previous histopathologic

\begin{tabular}{|c|c|c|c|c|c|}
\hline \multirow{2}{*}{$\begin{array}{l}\text { Table } 2 \\
\text { Gene set }\end{array}$} & \multicolumn{5}{|c|}{$\begin{array}{l}\text { Enrichment of oxidative phosphorylation pathway using permutation- } \\
\text { based approaches }\end{array}$} \\
\hline & Phenotype & $\begin{array}{l}\text { Enrichment } \\
\text { at } 1 \%\end{array}$ & $\begin{array}{l}\text { Enrichment } \\
\text { at } 5 \%\end{array}$ & $\begin{array}{l}\text { Enrichment } \\
\text { at } 10 \%\end{array}$ & GSEA \\
\hline \multirow[t]{3}{*}{ OXPHOS } & $\begin{array}{l}\text { Lacunar } \\
\text { Stroke }\end{array}$ & 0.07 & 0.27 & 0.43 & 0.05 \\
\hline & MLI/LA & $0.0017^{a}$ & 0.07 & 0.55 & 0.74 \\
\hline & ILI & 1 & 0.50 & 0.83 & 0.61 \\
\hline \multirow[t]{3}{*}{ Complex I } & $\begin{array}{l}\text { Lacunar } \\
\text { Stroke }\end{array}$ & 0.32 & - & - & - \\
\hline & MLI/LA & 0.07 & - & - & - \\
\hline & $\mathrm{ILI}$ & 1 & - & - & - \\
\hline \multirow[t]{3}{*}{ Complex II-III } & $\begin{array}{l}\text { Lacunar } \\
\text { Stroke }\end{array}$ & 0.14 & - & - & - \\
\hline & MLI/LA & 0.14 & - & - & - \\
\hline & ILI subtype & 1 & - & - & - \\
\hline \multirow[t]{3}{*}{ Complex IV } & $\begin{array}{l}\text { Lacunar } \\
\text { Stroke }\end{array}$ & 1 & - & - & - \\
\hline & MLI/LA & 0.16 & - & - & - \\
\hline & $\mathrm{ILI}$ & 1 & - & - & - \\
\hline \multirow[t]{3}{*}{ Complex V } & $\begin{array}{l}\text { Lacunar } \\
\text { Stroke }\end{array}$ & 0.12 & - & - & - \\
\hline & MLI/LA & 0.14 & - & - & - \\
\hline & $\mathrm{ILI}$ & 1 & - & - & - \\
\hline
\end{tabular}

Abbreviations: GSEA = gene-set enrichment analysis algorithm; ILI = isolated lacunar infarct; LA = leukoaraiosis; MLI = multiple lacunar infarcts; OXPHOS = oxidative phosphorylation pathway.

a Significant at $p<0.0042$. assessments has allowed the detection of novel associations between OXPHOS and MLI/LA. In addition, our results have implications for pathogenesis of small vessel disease as they imply that pathologic differences between lacunar stroke subtypes are due to distinct underlying pathophysiologic processes. Given that Biffi et al. ${ }^{20}$ further tested shared OXPHOS associations between ischemic stroke and Alzheimer disease, additional studies will be needed to determine whether the associations between OXPHOS genetic variation and MLI/LA stroke can extend to neurodegenerative disease as well, and whether OXPHOS abnormalities are specific to MLI/LA or influence other manifestations of cerebral small vessel disease such as enlarged perivascular spaces and microbleeds.

This study has several strengths. All lacunar stroke cases were MRI confirmed. All individuals, cases and controls, were genotyped on the same genome-wide association study array, which means that the results are less likely to be confounded by technical artifacts. Our study also has limitations. We were unable to obtain MRIs in the control population for this analysis. Population-based studies show that a subset of these are likely to have some degree of small vessel disease. This may have some effect on our results, with our results underestimating the true difference between cases and controls. Second, no adequately sized replication dataset with MRI-verified lacunar stroke is currently available to replicate our findings, so we were unable to independently validate our findings. Similarly, our analysis was limited to individuals of Caucasian ancestry from the United Kingdom. Extension of this analysis to other populations will be an important future development. Future studies will be needed to independently replicate our findings, and to identify specific causal variants and how they lead to OXPHOS dysfunction. Finally, we cannot identify whether specific cell types (i.e., endothelial cells, neurons, glia) are particularly affected by the OXPHOS variants tested in this analysis, which would be helpful in planning functional follow-up studies.

\section{AUTHOR CONTRIBUTIONS}

Dr. Matthew Traylor: drafting/revising the manuscript for content, study concept or design, analysis or interpretation of data, statistical analysis, accepts responsibility for conduct of research and final approval. Dr. Chris Anderson: drafting/revising the manuscript for content, study concept or design, analysis or interpretation of data, statistical analysis, accepts responsibility for conduct of research and final approval. Dr. Robert Hurford: drafting/revising the manuscript for content, analysis or interpretation of data, accepts responsibility for conduct of research and final approval. Dr. Steve Bevan: drafting/revising the manuscript for content, accepts responsibility for conduct of research and final approval. Prof. Hugh Markus: drafting/revising the manuscript for content, study concept or design, acquisition of data, study supervision or coordination, accepts responsibility for conduct of research and final approval. 


\section{ACKNOWLEDGMENT}

The authors thank the centers that collected samples for the Young Lacunar Stroke DNA Resource (see the supplementary acknowledgment).

\section{STUDY FUNDING}

Hugh Markus is supported by an NIHR Senior Investigator award. Hugh Markus and Steve Bevan are supported by the NIHR Cambridge University Hospitals Comprehensive Biomedical Research Centre. Collection of the UK Young Lacunar Stroke Resource was primarily supported by a Functional Genomics grant from the Wellcome Trust with additional support from the Stroke Association. Genotyping and MT were supported by a project grant from the Stroke Association (TSA 2013/01) Dr. Anderson is supported by NIH-NINDS K23 NS086873 and a Fellowship in Therapeutic Investigation sponsored by the Massachusetts General Hospital Department of Neurology and Biogen Idec, Inc.

\section{DISCLOSURE}

The authors report no disclosures relevant to the manuscript. Go to Neurology.org for full disclosures.

Received February 16, 2015. Accepted in final form September 8, 2015

\section{REFERENCES}

1. Martinez-Fernandez E, Gil-Peralta A, Garcia-Lozano R, et al. Mitochondrial disease and stroke. Stroke 2001;32: 2507-2510.

2. Anderson CD, Biffi A, Rahman R, et al. Common mitochondrial sequence variants in ischemic stroke. Ann Neurol 2011;69:471-480.

3. Anderson CD, Biffi A, Nalls MA, et al. Common variants within oxidative phosphorylation genes influence risk of ischemic stroke and intracerebral hemorrhage. Stroke 2013;44:612-619.

4. Wagner BK, Kitami T, Gilbert TJ, et al. Large-scale chemical dissection of mitochondrial function. Nat Biotechno 2008;26:343-351.

5. Morato L, Bertini E, Verrigni D, et al. Mitochondria dysfunction in central nervous system white matter disorders. Glia 2014;62:1878-1894

6. Fisher CM. Lacunar strokes and infarcts: a review. Neurology 1982;32:871-876.

7. Boiten J, Lodder J, Kessels F. Two clinically distinct lacunar infarct entities? A hypothesis. Stroke 1993;24: 652-656.

8. Bamford J, Sandercock P, Dennis M, Burn J, Warlow C. Classification and natural history of clinically identifiable subtypes of cerebral infarction. Lancet 1991;337:15211526

9. Adams HP Jr, Bendixen BH, Kappelle LJ, et al. Classification of subtype of acute ischemic stroke: definitions for use in a multicenter clinical trial: TOAST: Trial of Org 10172 in Acute Stroke Treatment. Stroke 1993;24:35-41.

10. Price AL, Patterson NJ, Plenge RM, Weinblatt ME, Shadick NA, Reich D. Principal components analysis corrects for stratification in genome-wide association studies. Nat Genet 2006;38:904-909.

11. Abecasis GR, Auton A, Brooks LD, et al. An integrated map of genetic variation from 1,092 human genomes. Nature 2012;491:56-65.

12. O'Connell J, Gurdasani D, Delaneau O, et al. A general approach for haplotype phasing across the full spectrum of relatedness. PLoS Genet 2014;10:e1004234.

13. Howie BN, Donnelly P, Marchini J. A flexible and accurate genotype imputation method for the next generation of genome-wide association studies. PLoS Genet 2009;5: e1000529.

14. Liu JZ, McRae AF, Nyholt DR, et al. A versatile genebased test for genome-wide association studies. Am J Hum Genet 2010;87:139-145.

15. Subramanian A, Tamayo P, Mootha VK, et al. Gene set enrichment analysis: a knowledge-based approach for interpreting genome-wide expression profiles. Proc Natl Acad Sci USA 2005;102:15545-15550.

16. Rui W, Guan L, Zhang F, Zhang W, Ding W. PMinduced oxidative stress increases adhesion molecules expression in human endothelial cells through the ERK/AKT/NF-kappaB-dependent pathway. J Appl Toxicol Epub 2015 Apr 15.

17. Doll DN, Hu H, Sun J, Lewis SE, Simpkins JW, Ren X. Mitochondrial crisis in cerebrovascular endothelial cells opens the blood-brain barrier. Stroke 2015;46:1681-1689.

18. El-Hattab AW, Hsu JW, Emrick LT, et al. Restoration of impaired nitric oxide production in MELAS syndrome with citrulline and arginine supplementation. Mol Genet Metab 2012;105:607-614.

19. Koga Y, Akita Y, Junko N, et al. Endothelial dysfunction in MELAS improved by l-arginine supplementation. Neurology 2006;66:1766-1769.

20. Biffi A, Sabuncu MR, Desikan RS, et al. Genetic variation of oxidative phosphorylation genes in stroke and $\mathrm{Alz}$ heimer's disease. Neurobiol Aging 2014;35:1956-1958.

\section{Subspecialty Alerts by E-mail!}

Customize your online journal experience by signing up for e-mail alerts related to your subspecialty or area of interest. Access this free service by visiting Neurology.org/site/subscriptions/etoc.xhtml or click on the "E-mail Alerts" link on the home page. An extensive list of subspecialties, methods, and study design choices will be available for you to choose from—allowing you priority alerts to cutting-edge research in your field! 


\section{Neurology}

\section{Oxidative phosphorylation and lacunar stroke: Genome-wide enrichment analysis of common variants}

Matthew Traylor, Christopher D. Anderson, Robert Hurford, et al.

Neurology 2016;86;141-145 Published Online before print December 16, 2015

DOI 10.1212/WNL.0000000000002260

\section{This information is current as of December 16, 2015}

\section{Updated Information \& Services}

Supplementary Material

\section{References}

Subspecialty Collections

Permissions \& Licensing

Reprints including high resolution figures, can be found at: http://n.neurology.org/content/86/2/141.full

Supplementary material can be found at: http://n.neurology.org/content/suppl/2015/12/16/WNL.0000000000002 260.DC1

This article cites 17 articles, 8 of which you can access for free at: http://n.neurology.org/content/86/2/141.full\#ref-list-1

This article, along with others on similar topics, appears in the following collection(s):

All Cerebrovascular disease/Stroke

http://n.neurology.org/cgi/collection/all_cerebrovascular_disease_strok e

Association studies in genetics

http://n.neurology.org/cgi/collection/association_studies_in_genetics Case control studies

http://n.neurology.org/cgi/collection/case_control_studies

MRI

http://n.neurology.org/cgi/collection/mri

Information about reproducing this article in parts (figures,tables) or in its entirety can be found online at:

http://www.neurology.org/about/about_the_journal\#permissions

Information about ordering reprints can be found online:

http://n.neurology.org/subscribers/advertise

Neurology ${ }^{\circledR}$ is the official journal of the American Academy of Neurology. Published continuously since 1951, it is now a weekly with 48 issues per year. Copyright (C 2015 American Academy of Neurology. All rights reserved. Print ISSN: 0028-3878. Online ISSN: 1526-632X.

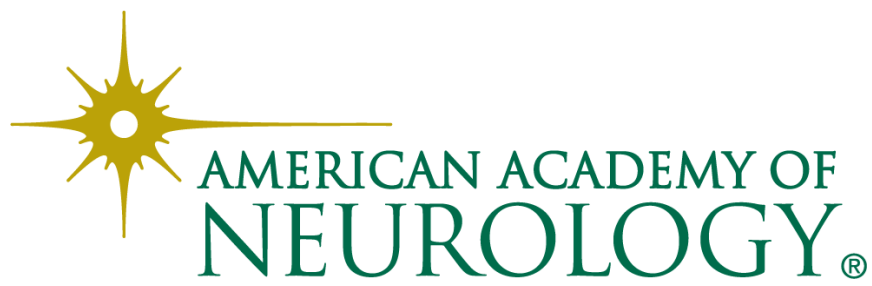

